\title{
TOBACCO HABITS AND ORAL HEALTH STATUS IN SELECTED INDIAN POPULATION
}

\author{
Sajith Vellappally ${ }^{1}$, Vimal Jacob ${ }^{1}$, Jindra Smejkalová ${ }^{1}$, Pilathadka Shriharsha ${ }^{2}$, Vinod Kumar ${ }^{3}$, Zdeněk Fiala $^{1}$ \\ ${ }^{1}$ Department of Hygiene and Preventive Medicine, Charles University in Prague, Faculty of Medicine in Hradec Králové, Czech Republic \\ 2Department of Dentistry, University Hospital in Hradec Králové, Czech Republic \\ ${ }^{3}$ Department of Oral Pathology and Microbiology, Amrito School of Dentistry, Kochi, India
}

\begin{abstract}
SUMMARY
This cross-sectional study was aimed at possible relationships between tobacco habits and selected behavior characteristics in an adult sample from India. Contemporaneous clinical examination comprised an intra-oral examination with specific emphasise to dental caries status in the form of DMFT (Decayed, Missing, and Filled Teeth) index. The study comprised 805 subjects in the age group from 30 to 69 years (72\% of males and $28 \%$ of females). The participants were divided into regular smokers, occasional smokers, ex-smokers, tobacco chewers and non-tobacco users. The highest prevalence of oral mucosal lesions were found in tobacco chewers $(22.7 \%)$ followed by regular smokers (12.9\%), occasional smokers $(8.6 \%)$, ex-smokers $(5.1 \%)$ and non tobacco users $(2.8 \%)(p<0.001)$. The mean number of decayed teeth was highest in tobacco chewers (6.96) followed by regular smokers (6.44) and ex-smokers $(5.5)(p<0.001)$. The mean number of missing teeth was highest in the group of regular smokers (1.9) and lowest in non-tobacco users (1.53), but the results were not statistically significant $(p=0.529)$. The mean number of filled teeth were highest in the group of tobacco chewers $(3.67)$ followed by regular smokers $(3.29)(p<0.001)$. DMFT value of tobacco chewers, regular smokers and ex-smokers is higher when compared to non-tobacco users $(p<0.001)$. The study documents that chewing tobacco and smoking can present significant risk factors for dental caries. However, the conclusions are burdened by some limitations. Further studies for investigation of the effect of tobacco using on dental caries are needed.
\end{abstract}

Key words: oral health, smoking, tobacco chewing, oral hygiene habits, DMFT, dental caries

Address for correspondence: Z. Fiala, Department of Hygiene and Preventive Medicine, Charles University, Faculty of Medicine, Šimkova 870, 50038 Hradec Králové, Czech Republic. E-mail: fiala@lfhk.cuni.cz

\section{INTRODUCTION}

In India tobacco consumption has been considered to be a major contributor to the total mortality rate and in $1990,1.5 \%$ of total deaths were tobacco related. Tobacco consumption is growing at the rate of $2-3 \%$ per annum (1). World Health Organization (WHO) assessment estimated that by 2020 tobacco related death may exceed 1.5 million annually or $13 \%$ of all deaths in India (2). India being a developing country and tobacco smoking is becoming the popular form of tobacco consumption in rural and urban population. Out of 930 million global tobacco consumers 1.1 billion smokers live in developing countries; with India alone has 182 million smokers (3).

In India, different forms of tobacco are being consumed. Cigarettes and bidis (hand rolled cigarettes that contain unprocessed tobacco) are the two most common forms of tobacco smoked in South India. Data reported by the Cancer Patients Aid Association of India in 2004 reveals that smoking of cigarettes (prevalence of $20 \%$ ) and bidis (prevalence of $40 \%$ ), followed by the use of smokeless tobacco (ST) in various forms (prevalence of $40 \%$ ) is widespread among both, men and women (1). The most common form of ST use is misri, a black powder obtained by roasting and powdering tobacco, which is then applied to the gum by using of fingers. Another most common form of ST is chewing of betel - quid, a combination of betel - leaves, areca nut, slaked lime, tobacco, and condiments; combination of ingredients is altered according to individual preferences. Documented data of Indian population suggested that $65 \%$ of all men and $33 \%$ of all women use some form of tobacco (1). However, demographic variation and distribution of forms of tobacco consumption in India are not uniform.

Major systemic adverse effects of tobacco consumption include various form of cancer (mainly lung cancer) and various forms of cardiovascular diseases. Likewise, there is strong evidence that tobacco use has numerous negative effects on oral health, for example, staining of teeth and dental restorations, reduction of the ability to smell and taste, development of oral diseases such as smokers' palate, smokers' melanosis and coated tongue. In addition, it seems that tobacco consumption can be partially related to oral pre-cancer, oral cancer, oral candidosis, periodontal disease, implant failure and dental caries $(4,5)$.

Even though dental caries has a multifactorial etiology (6) there is a suggestive relation between tobacco use and dental caries incidence. Unfortunately, only limited number of studies investigated relationships between the prevalence of dental caries and tobacco consumption (7). In recent years several studies 
indicated positive correlation between tobacco use and dental caries (8-13) but the conclusions are not wholly consistent and more studies are required to highlight this relation.

The general aim of presented cross sectional study was to contribute to better understanding of the relation between tobacco habits and oral health status. The specific aim was to investigate the existence (or non existence) correlation between tobacco consumption pattern and dental caries in randomly selected patients, who visited dental clinics in the South Indian city of Kochi, Kerala.

\section{MATERIAL AND METHODS}

\section{Subjects and Methods of Examination}

Studied group consisted of 805 adult patients (580 men and 295 women) aged between 30-69 years. The patients were examined in two private dental clinics in City of Kochi from March to July 2007. Cooperating dentists were instructed by the solvers of the study, to decrease the personal bias. The subjects in the age between of 30-69 were selected randomly, regardless of sex. The only exclusion criterion was age. Examinations consisted of two parts, a self-administered questionnaire and a clinical examination. The questionnaire contained items of the patients background information (age, sex, education), tobacco habits, eating and drinking habits, oral hygiene practices and participation in dental preventive check-ups. Clinical examination comprised an intraoral examination with specific emphasis to dental caries status in the form of DMFT (Decayed, Missing, and Filled Teeth) index.

\section{Questionnaire}

Personal data, education (no education, basic education, high school, graduation), oral health behavior and tobacco habits of patients were assessed by using a questionnaire.

Oral hygiene and oral health behavior was classified according to dental aids used for oral hygiene, brushing frequency, frequency of preventive dental visits and consumption of sweets, sweet drinks and alcoholic drinks. Dental aids comprised tooth brush and paste, tooth brush alone, tooth brush and tooth powder, dental floss, mouth wash, motorized tooth brush and tooth pick. Details of brushing frequency were recorded as once a day, two times a day, three times a day or never. Frequency of preventive dental visits was recorded as 2 times a year, once a year, visit only when having a problem or never before. Respondents were asked about the frequency of sweet, sweet drinks and alcohol consumption (daily, several times per week, several times per month, less frequently, never before).

Tobacco status of patients was classified into five groups: regular smoker, occasional smoker, ex-smoker, tobacco chewers and non-tobacco users. Regular smoker-smokes at least 1 cigarette/day, occasional smoker-smokes less than 1 cigarette/day, ex-smoker-smoked at least 1 cigarette per day for more than 6 consecutive months and now doesn't smoke at least for last 6 months, tobacco chewers-doesn't smoke but only chew tobacco and non-tobacco users never - used any form of tobacco (smoking or smokeless tobacco).

\section{Oral Examination}

As caries experience is an important index for assessing oral health awareness of adult, oral health examination were performed according WHO oral examination procedures (14). Oral examinations were performed after the questionnaire session and before dental treatment and in order to avoid any inference related to answer. Examiners were 5 dentists, who were able to examine with reasonable consistency, using a common standard. Four caries score were utilized, decayed teeth (reflecting the more recent untreated disease experience); missing teeth; filled teeth to estimate the caries treatment experience; and decayed, missing, and filled teeth (DMFT) all together to provide an estimate of the accumulated caries experience score. Each clinician carried out all clinical examination using artificial light, a flat surface mouth mirror, gauze, sponge dry air.

\section{Statistical Analysis}

Statistical analysis was performed by NCSS 2004 program. Mann-Whitney test and Kolmogorov-Smirnov test were used for comparing two groups of quantitative data. Kruskal-Wallis analysis of variance with multiple comparison tests was used for comparing of more groups. $\chi^{2}$-test of independence in contingency tables and Fisher's exact test was used for qualitative data. In all calculations the level of statistic significance was 0.05 .

\section{RESULTS}

\section{Tobacco Users and Non-tobacco Users}

The studied population was primarily classified into two groups as tobacco users, who used both smoking and chewing tobacco and non-tobacco users, who never used tobacco in any form. Classification of subjects according to the tobacco use by gender and age is shown in Table 1 . The differences were statistically significant. The majority of the respondents were male tobacco users. The mean age of the tobacco users was 47.29 and that of non-tobacco users, 40.31 years.

Table 1. Classification of respondents according to tobacco use and gender

\begin{tabular}{|l|c|c|c|c|c|}
\hline & $\begin{array}{c}\text { Males } \\
(\mathbf{n})\end{array}$ & $\begin{array}{c}\text { Females } \\
(\mathbf{n})\end{array}$ & $\begin{array}{c}\text { Total } \\
(\mathbf{n})\end{array}$ & $\begin{array}{c}\text { Age } \\
\text { (mean) }\end{array}$ & $\begin{array}{c}\text { Age } \\
\text { (SD) }\end{array}$ \\
\hline $\begin{array}{l}\text { Tobacco } \\
\text { users }\end{array}$ & 542 & 121 & 663 & 47.29 & 11.26 \\
\hline $\begin{array}{l}\text { Non-tobacco } \\
\text { users }\end{array}$ & 38 & 104 & 142 & 40.31 & 10.43 \\
\hline
\end{tabular}

Sex differences: $p<0.001 ; \chi^{2}$ test

Age differences: $p<0.001$, Kolmogorov-Smirnov test

\section{Tobacco and Smoking Status}

For detailed description, tobacco users were divided into four subgroups: regular smokers, occasional smokers, ex-smokers and tobacco chewers. Table 2 shows the detailed tobacco consumption history of males and females of studied population. One can see that regular smokers are mainly males (98\%) while in the subgroup of non-tobacco users were mainly females (73.2\%). It was 
Table 2. Characterization of respondents according the detailed tobacco consumption history

\begin{tabular}{|l|c|c|c|c|c|c|}
\hline & $\begin{array}{c}\text { Males } \\
(\mathbf{n})\end{array}$ & $\begin{array}{c}\text { Males } \\
(\%)\end{array}$ & $\begin{array}{c}\text { Females } \\
(\mathbf{n})\end{array}$ & $\begin{array}{c}\text { Females } \\
(\%)\end{array}$ & $\begin{array}{c}\text { Total } \\
(\mathbf{n})\end{array}$ & $\begin{array}{c}\text { Total } \\
(\%)\end{array}$ \\
\hline Regular smokers & 433 & 98.0 & 9 & 2.0 & 442 & 100.0 \\
\hline Occasional smokers & 33 & 94.3 & 2 & 5.7 & 35 & 100.0 \\
\hline Ex-smokers & 35 & 89.7 & 4 & 10.3 & 39 & 100.0 \\
\hline Tobacco chewers & 35 & 24.8 & 106 & 75.2 & 141 & 100.0 \\
\hline Non-tobacco users & 38 & 26.8 & 104 & 73.2 & 142 & 100.0 \\
\hline
\end{tabular}

$p<0.001 ; \chi^{2}$ test

Table 3. Respondents participating in preventive dental check-ups (\%)

\begin{tabular}{|l|c|c|c|c|}
\hline & Twice a year & Once a year & $\begin{array}{c}\text { Visits only when having } \\
\text { problem }\end{array}$ & Never before \\
\hline Regular smokers & 15.2 & 15.8 & 65.8 & 3.2 \\
\hline Occasional smokers & 2.9 & 25.7 & 60.0 & 11.4 \\
\hline Ex-smokers & 12.8 & 23.1 & 59.0 & 5.1 \\
\hline Tobacco chewers & 7.1 & 23.4 & 62.4 & 7.1 \\
\hline Non-tobacco users & 20.4 & 12.0 & 65.5 & 2.1 \\
\hline Total & 14.0 & 17.3 & 64.6 & 4.1 \\
\hline
\end{tabular}

$\mathrm{p}<0.001 ; \chi^{2}$ test

also found that in the subgroup of tobacco chewers, proportion of females was higher (75.2\%) compared to males (24.8\%). The results were statistically significant $(\mathrm{p}<0.001)$.

\section{Tobacco Status and Education}

We found that the educational level had significant influence on tobacco consumption and on the type of tobacco consumption. The highest frequencies of regular and occasional smokers as well as non tobacco users were found in the group of graduates (50.7 \%, $60.0 \%$ and $59.9 \%$ respectively). On the other hand in this group we found the lowest prevalence of tobacco chewers (37.6\%). For the lowest educated respondents we found the highest frequency of tobacco chewers $(10.6 \%)$. The results were statistically significant $\left(\chi^{2}\right.$-test; $\left.\mathrm{p}<0.05\right)$.

\section{Tobacco Status and Preventive Care}

Table 3 shows participation in preventive check-ups. Irrespective of tobacco consumption, $64.6 \%$ of respondents visited the dentist only when they had some dental problems. Only $14 \%$ of all the respondents participated in dental preventive checkup twice a year, among them mostly were non-tobacco users $(20.4 \%)$. The results are statistically significant $(\mathrm{p}<0.001)$.

\section{Tobacco Status and Oral Health Habits}

Most of the respondents brushed their teeth once a day (82.4\%), among them most of them being the regular smokers (87.8\%) (Table 4). Among those who brushed their teeth twice a day the regular smokers are less frequent (10.9\%). Three times a day brush their teeth only a few respondents, among them mostly
Table 4. Brushing frequency of tobacco consumers (\%)

\begin{tabular}{|l|c|c|c|c|c|c|}
\hline & \multicolumn{5}{|c|}{ Type of tobacco consumption } \\
\hline Brushing frequency & 1 & 2 & 3 & 4 & 5 & Total \\
\hline Once daily & 87.8 & 71.4 & 79.5 & 75.9 & 75.4 & 82.4 \\
\hline Twice daily & 10.9 & 22.9 & 20.5 & 22.7 & 20.4 & 15.6 \\
\hline Three times a day & 1.4 & 5.7 & 0.0 & 1.4 & 4.2 & 2.0 \\
\hline
\end{tabular}

$\mathrm{p}=0.0009 ; \chi^{2}$ test

1 - regular smokers, 2 - occasional smokers, 3 - ex-smokers, 4 - tobacco chewers, 5 - non-tobacco users

occasional smokers (5.7 \%) and non-tobacco users (4.2 \%). The results are statistically significant $(\mathrm{p}<0.001)$.

Table 5 shows the using of various dental aids by our respondents. Irrespective of tobacco consumption, majority of the respondents use tooth brush and paste to clean their teeth (90.9\%). The lowest frequency was found among tobacco chewers $(83.0 \%)$. The results are statically significant $(\mathrm{p}=0.009)$. Among our respondents tooth brush alone, tooth brush and powder and motorized tooth brush are not used so commonly. Dental floss was used among $5.3 \%$ of the whole study population and more frequently used by ex-smokers and non tobacco users $(12.8 \%$ and $12.7 \%$ respectively). Lowest use was seen among regular smokers $(3.4 \%)$ and tobacco chewers $(0.7 \%)(\mathrm{p}<0.001)$. Among those who used mouth wash, highest frequency was found in ex-smokers (20.3\%) and lowest frequency was found in tobacco 
Table 5. Use of various dental aids (\%)

\begin{tabular}{|l|c|c|c|c|c|c|c|}
\hline & \multicolumn{9}{|c|}{ Type of tobacco consumption } \\
\hline & 1 & 2 & 3 & 4 & 5 & Total & $p$ value \\
\hline Tooth brush and paste & 93.0 & 91.4 & 89.7 & 83.0 & 92.3 & 90.9 & 0.009 \\
\hline Tooth brush alone & 0.2 & 0.0 & 0.0 & 0.7 & 0.0 & 1.75 & 0.783 \\
\hline Tooth brush and powder & 4.1 & 2.9 & 10.3 & 7.8 & 6.3 & 5.4 & 0.229 \\
\hline Dental floss & 3.4 & 8.6 & 12.8 & 0.7 & 12.7 & 5.3 & $<0.001$ \\
\hline Mouth wash & 8.8 & 14.3 & 20.3 & 6.4 & 14.8 & 10.3 & 0.021 \\
\hline Motorized tooth brush & 1.1 & 0.0 & 0.0 & 0.7 & 2.1 & 1.1 & 0.680 \\
\hline Tooth pick & 14.0 & 20.0 & 41.0 & 23.4 & 11.3 & 16.8 & $<0.001$ \\
\hline
\end{tabular}

$\left(\chi^{2}\right.$ test)

1 -regular smokers , 2-occasional smokers , 3-ex-smoker , 4-tobacco chewers , 5 - non-tobacco users

chewers $(6.4 \%)(\mathrm{p}=0.021)$. Tooth picks are used mainly by exsmokers (41\%).

\section{Tobacco Status and Food and Drinks Habits}

Table 6 shows that the highest daily consumption of sweets and sweet drinks was found among regular smokers $(12.4 \%$ and $3.8 \%$ respectively) followed by tobacco chewers $(7.1 \%$ and $3.5 \%$ respectively). The results are statistically significant ( $p<0.001)$. We also found that a higher percentage of regular smokers consumed alcohol daily (3.8\%) followed by occasional smokers $(2.9 \%)$ and tobacco chewers $(2.8 \%)$ while a highest percentage of non-tobacco users (81.0\%) abstained from taking alcoholic drinks. The results are statistically significant $(\mathrm{p}<0.001)$.

\section{Tobacco Status and Oral Mucosal Lesions}

The highest prevalence of oral mucosal lesions were found in tobacco chewers (22.7\%) followed by regular smokers (12.9\%),

Table 6. Consumption of chosen food and drinks by the tobacco status groups (\%)

\begin{tabular}{|c|c|c|c|c|c|c|}
\hline & \multicolumn{6}{|c|}{ Type of tobacco consumption } \\
\hline & 1 & 2 & 3 & 4 & 5 & Total \\
\hline \multicolumn{7}{|c|}{ Sweets (e.g. cake, chocolate, biscuits) $p<0.001 ; \chi^{2}$ test } \\
\hline Never & 2.0 & 0.0 & 0.0 & 5.0 & 2.1 & 2.4 \\
\hline Less frequently & 47.1 & 45.7 & 51.3 & 59.6 & 54.9 & 50.8 \\
\hline S. t. per month & 16.7 & 22.9 & 38.5 & 19.9 & 27.5 & 20.5 \\
\hline S. t. per week & 21.1 & 31.4 & 5.1 & 8.5 & 12.7 & 17.4 \\
\hline Daily & 12.4 & 0.0 & 5.1 & 7.1 & 2.8 & 8.9 \\
\hline \multicolumn{7}{|c|}{ Sweet drinks (e.g. cola, juice) $p<0.001 ; \chi^{2}$ test } \\
\hline Never & 1.1 & 5.7 & 5.1 & 11.3 & 6.3 & 4.3 \\
\hline Less frequently & 29.9 & 37.1 & 46.2 & 42.6 & 38.0 & 34.7 \\
\hline S. t. per month & 40.5 & 28.6 & 28.2 & 29.8 & 44.4 & 38.2 \\
\hline S. t. per week & 24.7 & 28.6 & 20.5 & 12.8 & 10.6 & 20.0 \\
\hline Daily & 3.8 & 0.0 & 0.0 & 3.5 & 0.7 & 2.9 \\
\hline \multicolumn{7}{|c|}{ Alcoholic drinks (e.g. whisky, brandy, beer) $p<0.001 ; \chi^{2}$ test } \\
\hline Never & 10.2 & 40.0 & 59.0 & 70.9 & 81.0 & 37.2 \\
\hline Less frequently & 50.9 & 48.6 & 25.6 & 19.1 & 13.4 & 37.3 \\
\hline S. t. per month & 19.9 & 5.7 & 10.3 & 2.1 & 4.2 & 12.9 \\
\hline S. t. per week & 15.2 & 2.9 & 2.6 & 5.0 & 0.7 & 9.6 \\
\hline Daily & 3.8 & 2.9 & 2.6 & 2.8 & 0.7 & 3.0 \\
\hline
\end{tabular}

1 - regular smokers , 2 - occasional smokers , 3 - ex-smokers , 4 - tobacco chewers , 5 - non-tobacco users

S.t. - several times 
occasional smokers (8.6\%), ex-smokers (5.1\%) and non-tobacco users $(2.8 \%)$. The results were statically significant $\left(\chi^{2}\right.$ test; $\mathrm{p}<0.001)$.

\section{Tobacco Status and DMFT Index}

The mean number of decayed teeth (Table 7) was highest in tobacco chewers (6.96) followed by regular smokers (6.44) and ex-smokers (5.5). The results are statistically significant ( $\mathrm{p}<$ 0.001). The mean number of missing teeth (Table 8 ) was found to be highest in the group of regular smokers (1.9), followed by tobacco chewers (1.62) and ex-smokers (1.62). The lowest mean number of missing teeth was found in non-tobacco users (1.53). The results are not statistically significant $(\mathrm{p}=0.529)$. Table 9 shows that the mean number of filled teeth was highest in the group of tobacco chewers (3.67) followed by regular smokers (3.29). These results were statistically significant $(\mathrm{p}<0.001)$. The mean levels of collective DMFT values were: 12.25 (tobacco chewers), 11.63 (regular smokers), 10.35 (ex-smokers), 8.96 (nontobacco users) and 7.14 (occasional smokers) ( $<<0.001$ ).

\section{DISCUSSION}

The studied group is only a small sample of the Indian population. In India, adult males are expected to smoke and on the other hand females are not expected to smoke. This gender difference in the form of smoking behavior is clearly reflected in our study where $98 \%$ of regular male smokers were compared to only $2.0 \%$ of female regular smokers. Documented data show this similar trend in the whole Indian population. Only very few females smoke compared to the males (35\% males and 3\% females smoke), but both men and women use smokeless products to approximately the same extend $(2,15)$. One of the most important reasons for less female smoking in India is that traditional values do not favour smoking among young people and women. But there is no such taboo against using smokeless tobacco. Therefore, most women who use tobacco use it in smokeless forms. The study shows chewing tobacco use among women (75.2\%) is higher compared to men (24.8\%). Such trend was also seen in the study from large metropolitan city of Mumbai (16), which showed

Table 7. Tobacco status and the number of decayed teeth (DMF-D)

\begin{tabular}{|l|c|c|c|c|c|}
\hline & Regular smokers & Occasional smokers & Ex-smokers & Tobacco chewers & Non-tobacco users \\
\hline Mean & 6.44 & 3.6 & 5.5 & 6.96 & 5.1 \\
\hline SD & 3.95 & 2.67 & 3.78 & 4.44 & 4.25 \\
\hline Median & 6 & 4 & 4 & 6 & 4 \\
\hline 25th percentile & 3 & 2 & 3 & 4 & 2 \\
\hline 75th percentile & 8 & 5 & 9 & 10 & 6.25 \\
\hline
\end{tabular}

$\mathrm{p}<0.001$; Kruskal-Wallis One-Way ANOVA

Table 8. Tobacco status and the number of missing teeth (DMF-M)

\begin{tabular}{|l|c|c|c|c|c|}
\hline & Regular smokers & Occasional smokers & Ex-smokers & Tobacco chewers & Non-tobacco users \\
\hline Mean & 1.9 & 1.57 & 1.62 & 1.62 & 1.53 \\
\hline SD & 2.14 & 2.01 & 1.84 & 2.01 & 1.65 \\
\hline Median & 2 & 0 & 1 & 1 & 1 \\
\hline 25th percentile & 0 & 0 & 0 & 0 & 0 \\
\hline 75th percentile & 3 & 3 & 3 & 3 & 3 \\
\hline
\end{tabular}

$p=0.529 ;$ Kruskal-Wallis One-Way ANOVA

Table 9. Tobacco status and the number of filled teeth (DMF-F)

\begin{tabular}{|l|c|c|c|c|c|}
\hline & Regular smokers & Occasional smokers & Ex-smokers & Tobacco chewers & Non-tobacco users \\
\hline Mean & 3.29 & 1.97 & 3.23 & 3.67 & 2.33 \\
\hline SD & 3.2 & 2.20 & 3.09 & 3.61 & 2.86 \\
\hline Median & 2.5 & 2 & 2 & 3 & 2 \\
\hline 25th percentile & 1 & 0 & 1 & 1 & 0 \\
\hline 75th percentile & 5 & 3 & 5 & 5 & 3 \\
\hline
\end{tabular}

$\mathrm{p}<0.001$; Kruskal-Wallis One-Way ANOVA 
strong prevalence of women who use chewing tobacco (56\%). In many cultures particularly in the South-East Asia and increasingly in Sweden use of ST is more socially acceptable than smoking (17) and it is easy to practice it without detection or notice.

Tobacco consumption is often found to be disproportionately higher among lower socioeconomic groups $(3,18)$. However, barring a few local studies (19), little systemic investigation has been done into how tobacco consumption is socieconomically and geographically distributed in India. The study showed that higher percentage of people with higher education were non-tobacco users. It was also found that people, who are highly educated, mostly abstain from using chewing tobacco. This may be probably because chewing tobacco is cheaper compared to cigarette. Guthkas and Pan masala are popular type of chewing tobacco available in Indian market. They are sold in colorful small sachets for as low as half a rupee (ca US\$ 0.01) (€ 0.009) (15) and commonly used by people of lower socioeconomic class in India. The Indian national family health survey 1998-99 observed a strong gradient between education and chewing; the odds of chewing in educationally worst off group was 1.84 times higher that of people with post graduation (3) and thus sometimes becoming an issue of social status concern for the educated people not using chewing tobacco.

It was found that majority of the respondents visit dentist only when they have acute dental problem (see Table 3). Various studies have shown that socioeconomic and cultural factors can influence health by exposure to various physical and social environments $(20,21)$. Lack of use of preventive care may reflect a general attitude towards preventive care, difference in willingness or ability to pay for dental service or difference in the availability of dental care. The study also shows that the best participation in dental prevention had non-tobacco users, who participated on preventive check-ups most frequently. This is in agreement with other studies that smokers are generally less likely to pay regular preventive visits to dentists $(8,22,23)$.

We also found that lesser percentage of regular smokers brushes 2 times a day. This is also in agreement with other studies that non-smokers or non-tobacco users tend to brush their teeth more often than smokers or tobacco users who have poor brushing habit (24-27). From our study it resulted that dental aids like floss were more used by ex-smokers and non-tobacco users and less by regular smokers and tobacco chewers. Tobacco users are more reluctant to attend preventive dental care which with lesser frequency of brushing and lesser use of other dental aids can be associated with their bad oral hygiene. It shows tobacco users' careless attitude or less sophisticated outlook towards their general health care, particularly to oral health. This may result in periodontal problems and high caries risk in tobacco users. Our study reaffirmed the earlier reports that regular smokers show greater affinity towards high sugar contained products (7, $9,11)$ and alcohol $(7,23)$. Smokers tend to feel dry mouth more frequently than non-smokers $(9,28,29)$. Frequent intake of soft drink in smokers can be explained due to feeling of dry mouth. Our findings show that smokers and tobacco users in general exhibit undesirable behaviors with regard to health besides smoking and in agreement with other study reports (30).

In our study we found that those who were tobacco chewers (followed by regular smokers) had highest oral lesion compared with non-consumers. Similar results were also found in earlier studies from South Indian population (31). Oral mucosal changes or lesions may be due to local irritation or chronic stimulation of the lenfoid tissue in oral mucous membrane, caused by the use of tobacco.

The number of decayed and filled teeth in our study was highest in tobacco chewers in comparison with the other studied groups (see Table 7 and 9). DMFT value of tobacco chewers is higher when compared to non-tobacco users. This result can be compared to data from the multipurpose health survey (Third National Health and Nutrion Examination Survey) conducted in USA from 1988 to 1994 and done by Tomar et al. (13). A biologically reasonable explanation for an association between chewing tobacco use and dental caries may be the presence of high levels of fermentable sugar in chewing tobacco products, which can stimulate the growth of cariogenic bacteria. Locally prepared and commercially popular chewing tobacco products in India, like Pan masala and Guthkas, contains areca nut, tobacco, cardamon, lime and very often they are sweetened with unrefined sugar, sugar crystals, coconut or artificial sweeteners and flavors $(32,33)$. The added flavor and sweeteners are used to promote tobacco use and neutralize its harsh taste $(7,32,35)$. Chewing tobacco, typically used by placing a wad of tobacco between teeth and buccal mucosa, and is gently chewed or sucked over a period of several hours, is used over an extended period of each day $(7,13,35)$. This situation can create conducive environment for the initiation of dental caries by release of sugars from chewing tobacco to the local environment or oral cavity. Sugars in chewing tobacco can promote caries by bathing the teeth in cariogenic sugars $(7,13,36)$. This assumption is supported by in vitro evidence of stimulated growth of Streptococcus mutants and Streptococcus sanguis in the presence of smokeless tobacco extracts $(7,37)$.

Local loss of keratinized gingiva at the site where chewing tobacco is held can present another possible contributing mechanism in the development of mostly root-surface caries in tobacco chewers. This mechanism induces a tooth abrasion yielding periodontal problems reflecting the degree of gingival recession and bone loss. The exposed root surface, damaged by the loss of cementum and some dentin is at increased risk to develop caries (7).

The mean number of decayed, missing and filled teeth in our study was higher in regular smokers compared to non-tobacco users (see Tables 7-9). This is in agreement with other study results (11) where researchers reported that smoking as a habit positively correlated with increased number of decayed, missing and filled teeth. DMFT value of regular smokers and ex-smokers was higher when compared to non-tobacco users. The decreased buffering effect, possible lower $\mathrm{pH}$ of smoker's saliva and higher number of Lactobacilli and Streptococcus mutans group may indicate an increased susceptibility to caries in smokers (7). On the other hand, poor brushing habits, less use of dental aids and high sugar consumption among regular smokers (see Tables 4-6) would have contributed to increased caries incidence among our study sample (see Table 7).

The given statistics of tobacco status and missing teeth are not significant eventhough the trend from our study shows that the number of missing teeth is higher in regular smokers, followed by tobacco chewers and occasional smokers compared to nontobacco userss (see Table 8). Tobacco users in our sample showed poor oral hygiene and oral health habits. A previous study reported that combination of smoking and poor oral hygiene has a synergic 
effect on tooth loss (39). But it is most likely that smoking causes tooth loss through combined effect of periodontitis and dental caries, or joint effect of dental disease and behavioral or attitudinal factor (40). Tobacco use is associated with poor general health behaviors and it might also reflect a negative attitude towards the preservation of natural teeth. Neglect of caries treatment brings loss of teeth, what can explain why tobacco users have more missing teeth. Typical situation where tooth extraction is taken into consideration as a form of treatment are larger fracture, profound caries lesion with destruction of a large part of crown, acute pain or abscess caused by pulpal or periodontal involvement. These situations might be more frequent among tobacco users because of their unhealthy dental behaviors. A negative attitude to the preservation of natural teeth might lead to situation where smokers or tobacco users in general prefer tooth extraction instead of more conservative treatment. In Indian context, dental visits of the patients are self funded. Because of economic reasons and high cost of dental treatment, majority of dental visits are associated with acute dental problems like pain and infection etc. In India, there are no state supported preventive dental visits, mandatory for all citizens. An average age of first dental visit could be at adolescent period. Ongoing dental caries lesions were brought into the notice of patients and motivated for restorative dental treatments by the dentist. By that age there could be more dental caries lesions which require restorative treatments. This could be the possible reason for increased mean number of filled teeth among tobacco users (regular smokers, ex-smokers, tobacco chewers) (see Table 9).

The study exhibits some limitation that needs to be addressed. First of all, although general information about tobacco status was obtained, other information about tobacco usage including age of tobacco initiation and duration of tobacco usage was not taken. Further, study results were not representative of the Indian population. Our study group was a convenient sample and was not randomized. An exact comparison of our data with recent studies was not always possible because of differences in methodologies. Issues, such as dietary habits, oral habits, which have to be elucidated before any definite conclusions can be drawn regarding the effect of tobacco habits on development of dental caries.

\section{CONCLUSION}

Presented cross sectional study contributed to better understanding of relations between tobacco habits and oral health status. The study documents that chewing tobacco and smoking can present significant risk factors for dental caries. However, the conclusions are burdened by some limitations. Further studies for investigation of the effect of tobacco using on dental caries are needed.

\section{Acknowledgements}

This study was supported by the Department of Hygiene and Preventive Medicine, Faculty of Medicine, Hradec Králové, Charles University in Prague and by grant IGA MZ CR NR 8781-3/2006). The authors wish to thank Dr. Balachandar and Dr. Vinod Kumar, Kochi, India for supervising the fieldwork and collecting data and to RNDr. Eva Čermáková for data analysis.

\section{REFERENCES}

1. Chaly PE. Tobacco control in India. Indian J Dent Res. 2007 JanMar;18(1):2-5.

2. Rani M, Bonu S, Jha P, Nguyen SN, Jamjoum L. Tobacco use in India: prevalence and predictors of smoking and chewing in a national cross sectional household survey. Tob Control. 2003 Dec;12(4):e4.

3. Subramanian SV, Nandy S, Kelly M, Gordon D, Davey Smith G. Patterns and distribution of tobacco consumption in India: cross sectional multilevel evidence from the 1998-9 national family health survey. BMJ. 2004 Apr 3;328(7443):801-6.

4. Reibel J. Tobacco and oral diseases. Update on the evidence, with recommendations. Med Princ Pract. 2003;12 Suppl 1:22-32.

5. Vellappally S, Fiala Z, Smejkalová J, Jacob V, Somanathan R. Smoking related systemic and oral diseases. Acta Medica (Hradec Kralove). 2007;50(3):161-6.

6. Selwitz RH, Ismail AI, Pitts NB. Dental caries. Lancet. 2007 Jan 6;369(9555):51-9.

7. Vellappally S, Fiala Z, Šmejkalová J, Jacob V, Shriharsha P. Influence of tobacco use in dental caries development. Cent Eur J Public Health. 2007 Sep;15(3):116-21.

8. Locker D. Smoking and oral health in older adults. Can J Public Health. 1992 Nov-Dec; 83(6):429-32.

9. Axelsson P, Paulander J, Lindhe J. Relationship between smoking and dental status in 35-, 50-, 65-, and 75-year-old individuals. J Clin Periodontol. 1998 Apr;25(4):297-305.

10. Ludwick W, Massler M. Relation of dental caries experience and gingivitis to cigarette smoking in males 17 to 21 years old (at the Great Lakes Naval Training Center). J Dent Res. 1952 Jun;31(3):319-22.

11. Hirsch JM, Livian G, Edward S, Noren JG. Tobacco habits among teenagers in the city of Göteborg, Sweden, and possible association with dental caries. Swed Dent J. 1991;15(3):117-23.

12. Heng CK, Badner VM, Freeman KD. Relationship of cigarette smoking to dental caries in a population of female inmates. J Correctional Health Care. 2006 Jul 1;12(3):164-74.

13. Tomar SL, Winn DM. Chewing tobacco use and dental caries among U.S. men. J Am Dent Assoc. 1999 Nov;130(11):1601-10. Erratum in: J Am Dent Assoc. 1999 Dec;130(12):1700.

14. World Heath Organization. Oral hearth surveys: basic method. 4th ed. Geneva: WHO; 1997.

15. Shimkhada R, Peabody JW. Tobacco control in India. Bull World Health Organ. 2003;81(1):48-52.

16. Mackay J, Amos A. Women and tobacco. Respirology. 2003 Jun;8(2):12330 .

17. Mackay J, Eriksen M. The tobacco atlas. Geneva: WHO; 2002.

18. Giovino GA, Henningfield JE, Tomar SL, Escobedo LG, Slade J. Epidemiology of tobacco use and dependence. Epidemiol Rev. 1995;17(1):48-65.

19. Gupta PC. Survey of sociodemographic characteristics of tobacco use among 99,598 individuals in Bombay, India using handheld computers. Tob Control. 1996;5(2):114-20.

20. Power C, Hertzman C. Social and biological pathways linking early life and adult disease. Br Med Bull. 1997 Jan;53(1):210-21.

21. Adler NE, Boyce WT, Chesney MA, Folkman S, Syme SL. Socioeconomic inequalities in health. No easy solution. JAMA. 1993 Jun 2330;269(24):3140-5.

22. Millar WJ, Locker D. Smoking and oral health status. J Can Dent Assoc. 2007 Mar;73(2):155.

23. Telivuo M, Kallio P, Berg MA, Korhonen HJ, Murtomaa H. Smoking and oral health: a population survey in Finland. J Public Health Dent. 1995;55(3):133-8.

24. Alomari Q, Barrieshi-Nusair K, Said K. Smoking prevalence and its effect on dental health attitudes and behavior among dental students. Med Princ Pract. 2006;15(3):195-9.

25. Ainamo J. The seeming effect of tobacco consumption on the occurrence of periodontal disease and dental caries. Suom Hammaslaak Toim. 1971;67(2):87-94.

26. Kelbauskas E, Kelbauskiené S, Paipaliené P. Smoking and other factors influencing the oral health of Lithuanian Army recruits. Mil Med. 2005 Sep;170(9):791-6.

27. Macgregor ID. Survey of toothbrushing habits in smokers and nonsmokers. Clin Prev Dent. 1985 Nov-Dec;7(6):27-30.

28. Sakki TK, Knuuttila ML, Vimpari SS, Hartikainen MS. Association of lifestyle with periodontal health. Community Dent Oral Epidemiol. 1995 Jun;23(3):155-8. 
29. Ismail AI, Burt BA, Eklund SA. Epidemiologic patterns of smoking and periodontal disease in the United States. J Am Dent Assoc. 1983 May;106(5):617-21.

30. Thornton A, Lee P, Fry J. Differences between smokers, ex-smokers, passive smokers and non-smokers. J Clin Epidemiol. 1994 Oct;47(10): 1143-62.

31. Saraswathi TR, Ranganathan K, Shanmugam S, Sowmya R, Narasimhan PD, Gunaseelan R. Prevalence of oral lesions in relation to habits: cross-sectional study in South India. Indian J Dent Res. 2006 Jul-Sep; 17(3):121-5

32. Nair U, Bartsch H, Nair J. Alert for an epidemic of oral cancer due to use of the betel quid substitutes gutkha and pan masala: a review of agents and causative mechanisms. Mutagenesis. 2004 Jul;19(4):251-62.

33. Gupta PC, Ray CS. Epidemiology of betel quid usage. Ann Acad Med Singapore. 2004 Jul;33(4 Suppl):31-6.

34. Talhout R, Opperhuizen A, van Amsterdam JG. Sugars as tobacco ingredient: effects on mainstream smoke composition. Food Chem Toxicol. 2006 Nov;44(11):1789-98.

35. Zain RB, Ikeda N, Gupta PC, Warnakulasuriya S, van Wyk CW, Shrestha $\mathrm{P}$, et al. Oral mucosal lesions associated with betel quid, areca nut and tobacco chewing habits: consensus from a workshop held in Kuala Lumpur, Malaysia, November 25-27, 1996. J Oral Pathol Med. 1999 Jan;28(1):1-4.

36. Weintraub JA, Burt BA. Periodontal effects and dental caries associated with smokeless tobacco use. Public Health Rep. 1987 Jan-Feb; 102(1):30-5.

37. Lindemeyer RG, Baum RH, Hsu SC, Going RE. In vitro effect of tobacco on the growth of oral cariogenic streptococci. J Am Dent Assoc. 1981 Nov;103(5):719-22.

38. Zitterbart PA, Matranga LF, Christen AG, Park KK, Potter RH. Association between cigarette smoking and the prevalence of dental caries in adult males. Gen Dent. 1990 Nov-Dec;38(6):426-31.

39. Holm G. Smoking as an additional risk for tooth loss. J Periodontol. 1994 Nov;65(11):996-1001.

40. Ylöstalo P, Sakki T, Laitinen J, Järvelin MR, Knuuttila M. The relation of tobacco smoking to tooth loss among young adults. Eur J Oral Sci. 2004 Apr;112(2):121-6.

Received August 31, 2007 Accepted February 7, 2008 